\title{
Estudio comparativo de la evaluación a través de ejercicios sobre texto y vídeo para la identificación de elementos de una investigación científica
}

\author{
Daniel Cebrián Robles \\ dcebrian@uma.es \\ Departamento de Didáctica de las Ciencias Experimentales. Universidad de Málaga \\ Rafael Pérez-Galán \\ rpg@uma.es
}

Departamento de Didáctica y Organización Escolar. Universidad de Málaga.

\section{Natalia Quero-Torres}

natalia.quero@suubly.com

Departamento de Didáctica y Organización Escolar. Universidad de Málaga.

\begin{abstract}
Resumen
En la evaluación se utiliza cada vez más tecnologías como el vídeo digital por sus funcionalidades (anotaciones, ontologías y etiquetado social). La evaluación con anotaciones en el vídeo es una estrategia muy reciente e innovadora que ha sido poco investigada. Este trabajo pretende experimentar y evaluar el impacto de estas tecnologías emergentes en la formación inicial de docentes, comparando modelos tradicionales de evaluación en papel versus vídeo digital para evaluar competencias científicas en contenidos de vídeo que profusamente encontramos en las redes. Esta investigación responde a la pregunta, ¿existen diferencias en cuanto a cantidad y calidad de elementos científicos identificados por los estudiantes cuando se utilizan procedimientos de evaluación en papel versus vídeo digital? La muestra estaba formada por 31 estudiantes en formación inicial de maestros de infantil dentro de la asignatura de "Hacia una escuela inclusiva: modelos y prácticas". Como instrumento de análisis de las respuestas, se aplicó una rúbrica digital analizando 549 anotaciones y se utilizó la prueba estadística de Wilcoxon para la correlaciones. Se encontró que no había diferencias significativas en cuanto a la cantidad de elementos identificados, pero sí en cuanto a la calidad, siendo mejores las respuestas realizadas con el vídeo. El uso de la tecnología de las anotaciones sobre vídeo para actividades ha resultado eficiente para mejorar la calidad de las respuestas, pues obligaba a una detenida visualización del mismo, así como no tener que tomar notas mientras estaba la proyección del vídeo.
\end{abstract}

\section{Palabras clave}

Formación del profesorado; Video Digital; Anotaciones de Video; Rúbrica digital; Competencias Científicas 


\title{
Comparative study of assessment through text and video exercises for the identification of elements of a scientific investigation
}

\author{
Daniel Cebrián Robles \\ dcebrian@uma.es \\ Departamento de Didáctica de las Ciencias Experimentales. Universidad de Málaga \\ Rafael Pérez-Galán \\ rpg@uma.es \\ Departamento de Didáctica y Organización Escolar. Universidad de Málaga.

\section{Natalia Quero-Torres} \\ natalia.quero@suubly.com \\ Departamento de Didáctica y Organización Escolar. Universidad de Málaga.
}

\begin{abstract}
In the evaluation, technologies such as digital video are increasingly used for their functionalities (annotations, ontologies and social tagging). Assessment with video annotations is a new and innovative functionality that has been little researched. This work aims to experiment and evaluate the impact of these emerging technologies in the initial training of teachers, comparing traditional models of evaluation through the paper format versus digital video to assess scientific competences in video content that we find profusely in networks. This research answers the question: are there differences in the quantity and quality of scientific elements identified by students when using assessment procedures on paper versus digital video? The sample consisted of 31 pre-service teachers within the subject of "Towards an inclusive school: models and practices". As an instrument for the analysis of the responses, a digital rubric was applied analysing 549 annotations and the Wilcoxon statistical test was used for the correlations. It was found that there were no significant differences in the quantity of elements identified, but there were significant differences in the quality, with better responses being made with the video. The use of video annotation technology for activities has been efficient to improve the quality of the responses, as it forced a careful visualization of it, as well as not having to take notes while the video was being projected.
\end{abstract}

\section{Keywords}

Teacher Education; Digital Video; Video Annotations; e-Rubric; Scientific Competences. 


\section{Introducción}

Las modalidades de enseñanza están siendo revisadas con el desarrollo constante de las tecnologías y los cambios que estas producen en las prácticas educativas, que solicitan nuevas competencias a los docentes (Cabero-Almenara, Roig-Vila, \& Mengual-Andrés, 2018; GarcíaValcárcel \& Martín-del-Pozo, 2016), con procesos cada vez más digitalizados, y una enseñanza con espacios virtuales y semipresencial -b-learning- (Castaño-Garrido, Garay-Ruiz, \& Themistokleous, 2018; García-Ruiz, Aguaded, \& Bartolomé-Pina, 2018). Entre estas tecnologías, el vídeo digital es un recurso tradicional y actualmente en alza, que tiene una adecuada acogida por su buena usabilidad y las nuevas funcionalidades que permite su tecnología. A este auge se une el aumento considerable de contenidos en las plataformas en internet (Youtube y Vimeo) como en los repositorios y portales de recursos institucionales, donde se comparten un importante número de contenidos para diferentes áreas educativas, y donde se exponen las ventajas de su utilización para la educación en general (Bryan \& Recesso, 2006; Marques \& Reis, 2017). A pesar del crecimiento y presencia de la tecnología del video en la sociedad y en las prácticas de los centros educativos, necesitamos experimentar y evaluar los últimos avances de la tecnología del vídeo (anotaciones, ontología y etiquetado) en la formación inicial y permanente de los docentes, pues si se reconocen estas ventajas y buenas prácticas escolares, pocos estudios analizan el compromiso de los docentes en el uso de los vídeos en programas de desarrollo profesional (Mirriahi, Jovanovic, Dawson, Gašević, \& Pardo, 2018). No existen suficientes experiencias en la evaluación de los aprendizajes con las tecnologías del vídeo digital (Cebrián-de-la-Serna, Bartolomé-Pina, CebriánRobles, \& Ruiz-Torres, 2015). No investigar en esta línea sorprendería si aceptáramos la conocida sentencia "enseñarás como te enseñaron", y comprobaríamos los resultados positivos que encontraríamos en la literatura especializada sobre el vídeo digital y las anotaciones multimedia en su impacto sobre las competencias reflexivas en educación, las competencias científicas y matemáticas en la formación inicial y permanente de docentes (Barth-Cohen, Little, \& Abrahamson, 2018; Mirriahi et al., 2018).

La reflexión sobre la propia enseñanza es un contenido elemental en la formación inicial y permanente de los docentes (Montero \& Gewerc, 2018), que requiere de capacitación para emplear los mecanismos de racionalidad en el análisis, que con la formación en competencia digital (AlonsoFerreiro, 2018) debería llevarles a ser un docentes "transformadores" y comprometidos con el cambio (Cela-Ranilla, Esteve González, Esteve Mon, González Martínez, \& Gisbert-Cervera, 2017). El vídeo se ha visto pertinente para facilitar el análisis para el cambio sobre los modelos y prácticas innovadoras (Cebrián-Robles, Cebrián-de-la-Serna, \& Monedero-Moya, 2015). No obstante, se ha comprobado que los estudiantes en formación inicial tienen una falta de conocimiento en cuanto al planteamiento científico y, sobre todo, cómo realizar una indagación científica y la creación y diseño de unidades didácticas basadas en la indagación (Toma, Greca, \& Meneses-Villagrá, 2017).

\section{a. El uso educativo de las anotaciones en el vídeo}

Los resultados de diversas investigaciones muestran que el vídeo es una única y poderosa herramienta educativa (Gaudin \& Chaliès, 2015). Con el surgimiento de los vídeos digitales han aumentado nuevas funcionalidades como las de crear comentarios o "anotaciones" en el mismo vídeo, permitiendo interesantes oportunidades para analizar y desarrollar diferentes metodologías; así como favorecer el aprendizaje más colaborativo compartiendo experiencias y comentarios en comunidades de aprendizaje (Chiu et al., 2016). Con las funcionalidades de etiquetado social y la visibilidad que disponen las herramientas dentro de estas plataformas de vídeo (nubes de palabras, estadísticos, gráficos de los tags, etc) se convierte en una herramienta ideal para estudiar los contenidos educativos, y analizar la producción de anotaciones y comunicación interactiva que generan las anotaciones. 
Desde las consideraciones de Cebrián-Robles, Blanco-López y Noguera-Valdemar (2016), existen diferentes razones que hacen atractivo el uso de los vídeos y anotaciones en educación en general, y para todas las áreas de conocimiento, entre las que destacamos la cada vez mayor relevancia de los códigos multimedia en la comunicación humana, en la sociedad del conocimiento y en el mundo científico (Hernandez-Ramos, 2007; Santagata \& Guarino, 2011). Así se observa en la continua presencia de video artículos, vídeo-comunicaciones y vídeo-póster como formato de producción científica (Bozdag, 2008). Las evidencias de aprendizaje más fáciles de documentar con una cercana comunicación entre quienes enseñan y aprenden (Dovigo, 2016). Y porque hay competencias que requieren para su análisis evidencias multimedia (Cebrián-de-la-Serna et al., 2015).

Recientemente el video digital ha mostrado un punto de inflexión en cuanto a versatilidad y desarrollo en diferentes contextos educativos, como los producidos recientemente por dotar de mayor interactividad a los vídeos de los cursos en plataformas MOOC. Todo ello, en un intento de buscar soluciones a los problemas de importante abandono de los usuarios en estos formatos (Bozdag, 2008; Cebrián-Robles et al., 2015). Por tanto, se puede afirmar, desde la perspectiva de Cebrián-Robles et al. (2016), que se trata de una tecnología que utiliza componentes de código libre que pueden ser integrados en cualquier plataforma educativa como Moodle, WordPress, o como en nuestro proyecto de Coannotation, que es una plataforma creada específicamente para poder enlazar vídeos de la red y crear anotaciones sobre él. La versatilidad de Coannotation radica en las "anotaciones multimedia" de textos, imágenes, direcciones de internet, sonidos, etc. Además de etiquetar con palabras predefinidas (por el docente o usuarios), permite ver y analizar una gran variedad de aspectos, fundamentalmente la dispersión de las respuestas de los estudiantes en torno a una pregunta concreta (ver figura 1 ).

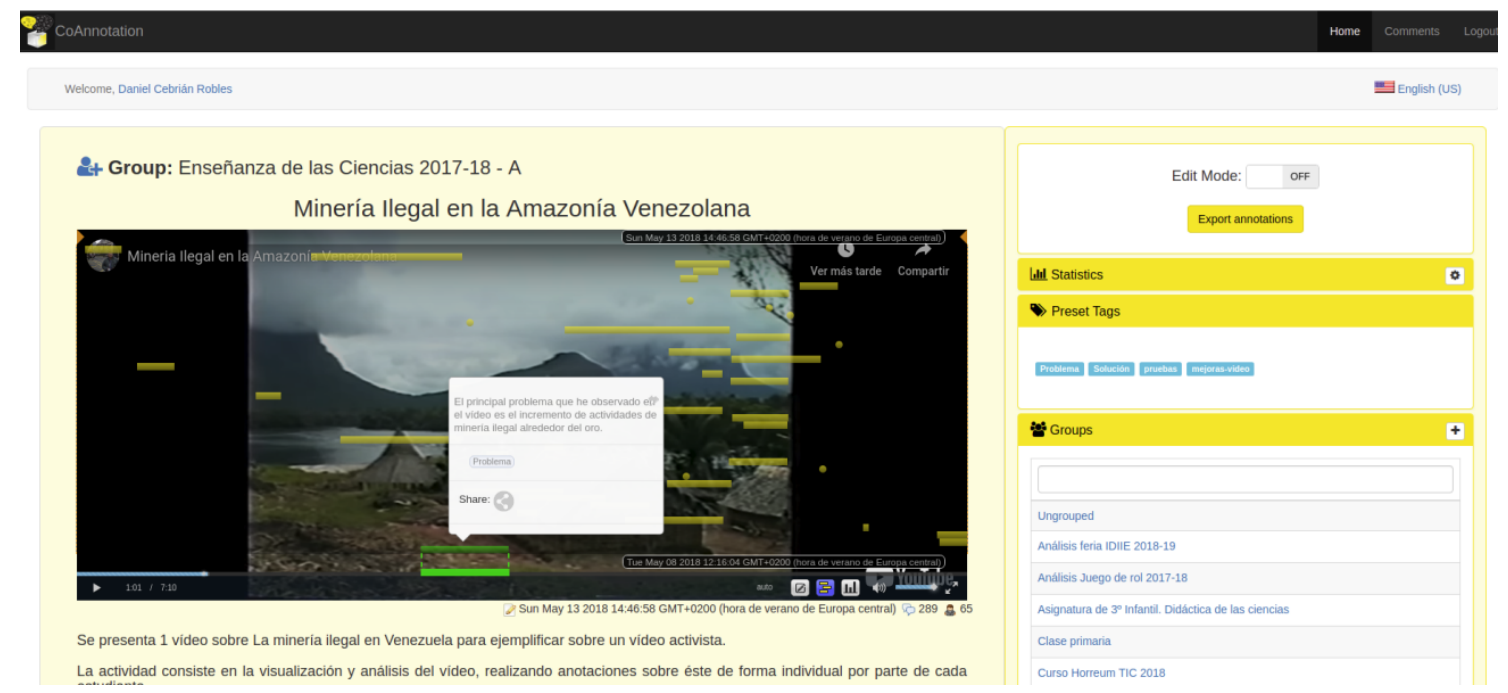

Figura 1. Captura de pantalla de Coannotation

Fuente: Elaboración propia

El uso de esta herramienta, por tanto, facilita que el alumnado sea capaz de identificar, proponer, definir, valorar aquellas secuencias de aprendizaje que a través de un modelo convencional se quedarían tan solo en una interpretación más efímera de la realidad objeto de estudio. Así, la implicación del alumnado en la tarea es muy activa porque el diseño de la propia actividad le confiere mecanismos de verificación mucho más atractivos: concreción, análisis, síntesis, evaluación... todos procesos metacognitivos favorecedores de un aprendizaje mucho más relevante, personal, crítico y, sobre todo, científico. 


\section{b. La indagación y la argumentación científica}

Las pequeñas investigaciones escolares o indagaciones son unos de los contenidos que se recogen en el currículo de infantil y primaria que fomentan el aprendizaje significativo. Son una manera de conectar a los niños y niñas con la realidad y así fomentar el interés y la curiosidad de forma natural (Vega, 2011). Minner, Levy \& Century (2010) después de una revisión de 138 artículos sobre la enseñanza científica en estudiantes de edades entre los 4 y 16 años, demuestran que existe una tendencia clara y consistente en la aplicación de la indagación escolar (mediante preguntas, diseño de experimentos, recopilación de datos...) como estrategia de enseñanza, y que ello provoca una mejora del aprendizaje y retención de los conocimientos científicos por parte del alumnado. Es por ello que, los estudiantes de los grados de educación deberían conocer cuál es el método científico y a partir de este, preparar pequeñas indagaciones para realizar en el aula. Se necesita más investigación sobre el impacto que tienen las estrategias formativas y las oportunidades de aprendizaje que mejoren en los estudiantes, en formación inicial de maestros, la toma de decisiones para indagar (Forbes \& Davis, 2010), pero antes es necesario que estos estudiantes conozcan cuáles son los elementos que componen una indagación. La literatura señala una falta de conocimiento, por parte de estos estudiantes en formación inicial, en cuanto a cómo realizar una indagación escolar y la identificación de los elementos que la componen (VílchezGonzález \& Bravo-Torija, 2015).

La argumentación científica es junto a la indagación uno de los ejes fundamentales de la enseñanza de las ciencias (Driver et al., 2000), pero también uno de los más complejos de analizar, ya que no existe consenso a la hora de analizar a través de un solo modelo la calidad lógica, de contenido y dialéctica de los argumentos. De acuerdo con Sadler (2011), las actuales herramientas para evaluar la calidad de la argumentación, ya sea con propósitos investigativos o propósitos relacionados con el aprendizaje, son limitadas. No obstante, entre los esquemas analíticos de argumentos, es bien conocido el modelo de Toulmin (1958), en cuanto paradigma convencional y clásico, que nos acerca en un primer momento a la conceptualización y uso de las argumentaciones científicas. La concepción de argumento de este autor ha permitido a los investigadores examinar la calidad de los argumentos en una gran variedad de dominios, entre los cuales se encuentra la ciencia (Jiménez-Aleixandre, Rodriguez, \& Duschl, 2000; Osborne, Erduran, \& Simon, 2004). Entre los elementos de los argumentos seguidos en el modelo de Toulmin (1958), caben destacar los elementos esenciales de un argumento, entre los que podemos encontrar: las afirmaciones o conclusiones a la que puede llegar una investigación; las pruebas o evidencias que soportan estas afirmaciones; y la justificación entendida como el nexo de unión de estas pruebas y las afirmaciones (Jiménez-Aleixandre, 2010). Existen serias dificultades a la hora de interpretar y definir correctamente qué se entiende por una adecuada argumentación con respecto al objeto de estudio y cuáles deben ser los elementos integradores de los argumentos, como su buen uso y desarrollo. Algunos programas formativos muestran diferentes actividades secuenciadas para mejorar la evaluación y construcción de argumentos por estudiantes en formación inicial de maestros (Cebrián-Robles, Franco-Mariscal, y Blanco-López, 2018). Según Osborne et al (2016), en el proceso de la argumentación científica no sólo existe la producción de argumentos, sino también la crítica o identificación de los elementos de estos.

\section{Objetivos}

Este trabajo persigue, tras la proyección de un vídeo documental de una investigación científica, experimentar y evaluar el impacto de la tecnología de las anotaciones de vídeo en la formación inicial de docentes, comparando modelos tradicionales de evaluación en papel versus vídeo digital para evaluar competencias científicas. Para ello se plantea la siguiente pregunta de investigación: ¿existen diferencias en cuanto a cantidad y calidad de elementos científicos identificados por los 
estudiantes cuando se utilizan procedimientos de evaluación en papel versus vídeo digital?

\section{Metodología}

Se seleccionó un grupo de 31 estudiantes en formación inicial de maestros dentro de la asignatura de formación básica, "Hacia una escuela inclusiva: modelos y prácticas" en la Fundación María Inmaculada de Antequera, adscrita a la Universidad de Málaga. La actividad se realizó con una proyección de un vídeo a todo el grupo, recomendando que tomaran notas a papel durante el visionado. Después de esta exposición se pasó una actividad de evaluación con unas cuestiones donde se solicitaba la identificación de los elementos (preguntas de investigación, hipótesis, pruebas, justificación y conclusiones) de la investigación científica observada en el vídeo. Se dividió el grupo de estudiantes en dos subgrupos, en uno se realizó esta prueba de evaluación contestando desde sus propias notas escritas y en un formato de papel, exclusivamente. Siendo esta modalidad una práctica muy frecuente en los centros educativos, donde los estudiantes tras una proyección de video realizan actividades de papel, sin que ello elimine la posibilidad de volver a consultar el contenido del vídeo. El otro subgrupo contestó a las mismas preguntas y con sus notas de papel, pero en un formato de anotaciones multimedia dentro del mismo vídeo. Quedando como variable independiente las diferencias en cuanto al formato de respuesta de la actividad de evaluación. La variable dependiente fue la cantidad y calidad de las respuestas sobre los elementos identificados en el vídeo, y que se plantearon en la actividad de evaluación. Se repitió el diseño de la experimentación de un primer vídeo con otro segundo siguiendo los mismos procedimientos, con la misma división de estudiantes y sin ninguna retroalimentación o evaluación por parte del docente en cuanto a sus respuestas.

\section{a. Vídeos sobre investigaciones científicas}

En la actividad se proyectaron dos vídeos de dos científicos diferentes Thorndike y Tinbergen. Sin embargo, en el vídeo de Thorndike se presentaron dos experiencias sobre las respuestas de los animales hacia diferentes estímulos para conocer la capacidad de aprendizaje. En el video de Tinbergen sólo se narraba una experiencia de investigación del motivo de la curiosa danza en zigzag del pez espinoso macho.

\section{a.a El aprendizaje de Thorndike}

El vídeo del aprendizaje de Thorndike ${ }^{1}$ (1898) consistió en un experimento científico en el que el autor quería conocer la capacidad de aprendizaje de los humanos, aunque usaba animales en sustitución, buscando las leyes generales del aprendizaje. Para ello, utilizó pruebas para ver la conducta, en diferentes animales, según diferentes estímulos. El vídeo constaba de dos experimentos de diferente naturaleza. El primero de ellos, realizado con pollos (de ahora en adelante llamado, experimento con pollos o EP), en el que se quería conocer la naturaleza del aprendizaje (Thorndike, 1898, p. 34). Para ello, utilizó un laberinto en donde se encerraron a los pollos para ver el tiempo que tardaban en salir, y mediante la repetición consecutiva de intentos se conocía la naturaleza progresiva del aprendizaje de los pollos. En el segundo vídeo, Thorndike experimentó el tiempo que tardaba un gato en aprender una nueva habilidad motivada por una recompensa (de ahora en adelante Ilamado, experimento con gatos o EG) (Thorndike, 1898, p. 12). Para ello, encerró al animal en una jaula que tenía una manivela para poder salir de ella y obtener el alimento. Tras sucesivos intentos, el gato iba aprendiendo la habilidad conforme más veces repetía la acción. Se concluyó en el vídeo que la conducta cambiaba a causa de sus consecuencias. 


\section{a.b La danza del pez espinoso}

El vídeo sobre la danza del pez espinoso ${ }^{2}$ presentó la experiencia de Tinbergen (1952) y su investigación sobre el comportamiento de la curiosa danza que realizaba los peces espinosos machos en época de cortejo (de ahora en adelante llamado, experimento con el pez espinoso o EE). La experiencia consistió en la preparación de diferentes modelos de cera que utilizaba en un acuario donde habían peces macho. Estos modelos de cera tenían tres formas diferentes: la del pez espinoso en su estado normal; la de la silueta de la hembra con la barriga cargada de huevos; y la del color rojizo de otro macho en celo. De la experiencia se dedujo que la aparición de la danza en zigzag se producía en mayor medida por la presencia de la hembra cargada de huevos, y esta era su técnica de reclamo para llevar a la hembra al nido donde depositaría sus huevos para ser fecundados. Sin embargo, un estado de agresividad aparecía cuando otro pez con el vientre color rojizo simulaba un macho en celo.

\section{b. Recogida de datos}

Como ya se ha explicado, la recogida de datos se realizó mediante dos formatos diferentes: el primero, un cuestionario a papel (para el grupo de control); y el segundo, la plataforma de anotaciones de vídeo coannotation.com (para el grupo experimental).

\section{b.a Cuestionario a papel}

Este cuestionario consistió en cinco preguntas abiertas, para conocer la cantidad de elementos del vídeo, y la adecuación de esta identificación a la pregunta que se le formulaba. Los enunciados de las preguntas fueron los siguientes: 1) Enumera todas las preguntas de investigación que se plantean en el vídeo; 2) Enumera todas las pruebas de investigación que aparecen en el vídeo; 3) Enumera todas las hipótesis que plantea el vídeo; 4) Enumera todas las conclusiones a las que llega el narrador del vídeo; 5) Enumera todas las justificaciones que plantea el científico en el vídeo.

\section{b.b Plataforma CoAnnotation.com}

La plataforma coannotation.com se usa como herramienta innovadora para la recogida de respuestas de los estudiantes. Para ello, se le indica a los estudiantes que tienen que identificar una serie de cuestiones que deben de buscar en el vídeo, las mismas que se le preguntan a los estudiantes del grupo de control, pero que en este caso tienen que hacerlo seleccionando fragmentos de vídeo para identificar y escribir con sus palabras cuáles son: las diferentes preguntas de investigación; las pruebas científicas; las hipótesis que se plantean en el vídeo; las conclusiones a las que llega el narrador; y la justificaciones que plantea el científico.

\section{c. Análisis de los datos}

El análisis de los datos se realizó de igual forma para los dos formatos a papel y mediante las anotaciones de vídeo. Las respuestas de los estudiantes son consideradas como adecuadas siempre que se acerquen a las esperadas por el profesorado. En la siguiente tabla 1 se aportan las respuestas más adecuadas según los autores del trabajo: 


\begin{tabular}{|c|c|}
\hline Elementos & Respuesta adecuada \\
\hline $\begin{array}{l}\text { Preguntas de } \\
\text { investigación }\end{array}$ & $\begin{array}{l}\text { EP: ¿Cuánto tiempo tarda un pollo en volver a aprender a salir de un laberinto y volver con sus } \\
\text { compañeros? o ¿Cuáles eran las leyes del aprendizaje? } \\
\text { EG: ¿Cómo pueden los animales aprender una nueva habilidad? } \\
\text { EE: ¿Cuáles eran los signos que le llevaban al macho a hacer la danza en zigzag o atacar? }\end{array}$ \\
\hline $\begin{array}{l}\text { Pruebas de } \\
\text { investigación }\end{array}$ & $\begin{array}{l}\text { EP: El tiempo que tardaron los pollos en salir del laberinto } \\
\text { EG 1: No detectó signos de intuición la primera vez que tenía que salir de la caja } \\
\text { EG 2: Gráficos para medir el índice del aprendizaje de una nueva habilidad }\end{array}$ \\
\hline Hipótesis & $\begin{array}{l}\text { EP: Los pollos aprenden más rápido al salir del laberinto con la práctica } \\
\text { EG: Si una acción tiene un premio, ésta supondría un aprendizaje al quedar grabado en la mente } \\
\text { EE. La danza en zigzag, parecía un reglamentado intercambio de señales (el cortejo). Los peces machos } \\
\text { se ponen frenéticos cuando ven algo de color rojo, como puede ser el camión de correos, al pensar que es } \\
\text { el vientre de un macho }\end{array}$ \\
\hline Conclusiones & $\begin{array}{l}\text { EP: Los resultados con los pollos permitieron conocer la naturaleza progresiva del aprendizaje } \\
\text { EG: La aparente inteligencia para aprender una habilidad, aparece por tanteos para obtener un premio, en } \\
\text { este caso comida. La conducta cambia a causa de sus consecuencias o ley de efecto que explica cómo } \\
\text { nuevos animales desarrollan nuevos hábitos } \\
\text { EE: El pez espinoso tiene una danza de cortejo cuando ve a una hembra cargada de huevos }\end{array}$ \\
\hline Justificaciones & $\begin{array}{l}\text { EP: Los sucesivos resultados de los tiempos que tardaban los pollos en salir del laberinto fueron menores } \\
\text { conforme repetían la experiencia, y esto daba una idea de la naturaleza progresiva del aprendizaje } \\
\text { EG: Las recompensas hacen que los animales cambien de hábitos por tanteos para sacar el mejor } \\
\text { provecho de cualquier situación } \\
\text { EE: El macho no presenta la danza en zigzag cuando se encuentra con un modelo parecido a un macho ni } \\
\text { cuándo se introducen un pez no embarazada. Sin embargo, sí que presenta la danza cuando se introduce a } \\
\text { una hembra en celo, por lo que el modelo influye en la danza de cortejol }\end{array}$ \\
\hline
\end{tabular}

Tabla 1. Respuestas adecuadas según los autores a las preguntas de la actividad. EP: experimento con pollos; EG: experimento con gatos; EE: experimento con el pez espinoso.

Fuente: Elaboración propia.

Para la evaluación de la actividad, se muestra una rúbrica, en la figura 2, creada en consenso por los autores del trabajo.

Se aceptará como elemento identificado en vídeo o en papel de forma adecuada, cuando éste sea similar a la respuesta adecuada de la tabla 1. 


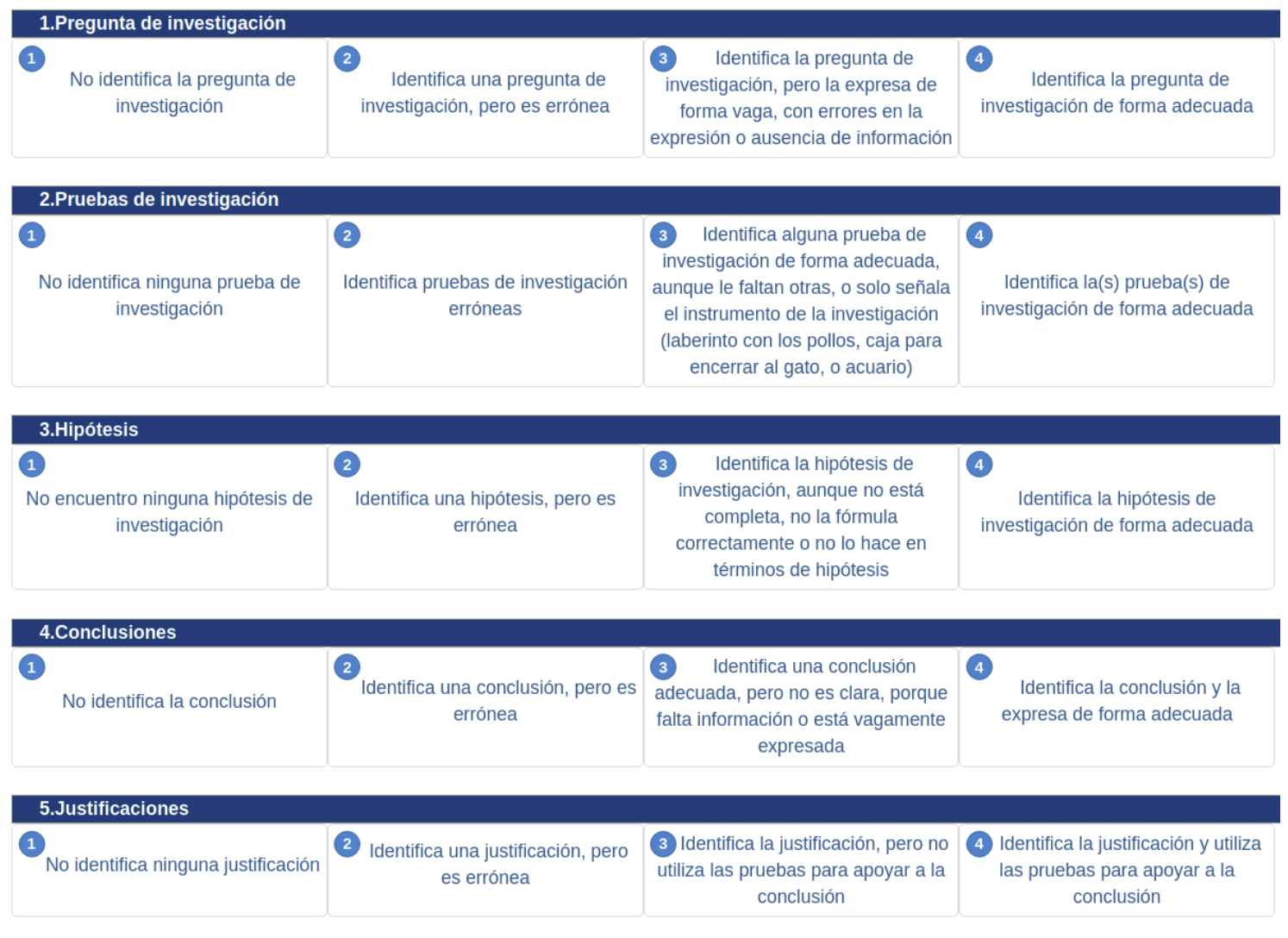

Figura 2. Rúbrica de evaluación de las preguntas de la actividad. Fuente: Elaboración propia

En ella, el primer nivel es la ausencia del elemento en cuestión, el nivel 2 es la identificación errónea del elemento, en el nivel 3 aunque se identifica el elemento de forma adecuada, no es expresada en términos científicos, de forma taxativa, o no apoya a la conclusión.

\section{Resultados}

Se obtuvieron (549 anotaciones) de los estudiantes. Los resultados del trabajo se muestran en la tabla 2 para cada uno de los elementos analizados en este trabajo (preguntas, pruebas, hipótesis, conclusiones y justificaciones). Estos fueron analizados para cada uno de las tres experiencias científicas y se compararon los resultados en formato papel y en formato vídeo para cuantificar el número de elementos encontrados y la calidad con la que el estudiante explicaba el elemento, teniendo en cuenta la rúbrica de la figura 2. 


\begin{tabular}{|c|c|c|c|c|c|c|c|c|c|}
\hline & & \multicolumn{4}{|c|}{ Número de identificaciones } & \multicolumn{4}{|c|}{ Nivel en la rúbrica } \\
\hline & & en papel & en vídeo & & & en papel & en vídeo & & \\
\hline & & x(Mdn) & x (Mdn) & $\mathrm{Z}$ & $p$ & x (Mdn) & x (Mdn) & $\mathrm{Z}$ & $p$ \\
\hline \multirow[t]{5}{*}{ Gatos } & Preguntas & $0.61(1)$ & $0.61(1)$ & -0.243 & NS & $2.03(2)$ & $2.68(4)$ & -2.08 & 0.037 \\
\hline & Pruebas & 0.90 (1) & 0.94 (1) & -0.299 & NS & $2.10(2)$ & 2.55 (3) & -2.181 & 0.029 \\
\hline & Hipótesis & $1.03(1)$ & $0.87(1)$ & -0.808 & NS & $2.35(2)$ & $2.32(2)$ & -0.088 & NS \\
\hline & Conclusiones & 1.35 (1) & $2.00(1)$ & -1.715 & NS & $2.55(2)$ & $3.10(4)$ & -2.087 & 0.037 \\
\hline & Justificaciones & $0.90(1)$ & $0.84(1)$ & -0.234 & NS & $2.10(2)$ & $2.10(2)$ & -0.021 & NS \\
\hline \multirow[t]{5}{*}{ Pez } & Preguntas & $1.22(1)$ & $1.56(1)$ & -0.595 & NS & $2.56(2)$ & $2.83(3)$ & -0.637 & NS \\
\hline & Pruebas & $1.44(1)$ & $1.83(1)$ & -0.873 & NS & $2.50(2)$ & $2.94(3)$ & -1.812 & NS \\
\hline & Hipótesis & $1.00(1)$ & $1.06(1)$ & -0.104 & NS & $2.11(2)$ & $2.17(2)$ & -0.184 & NS \\
\hline & Conclusiones & 0.78 (1) & $1.11(1)$ & -1.265 & NS & $1.89(2)$ & $2.22(2)$ & -1.403 & NS \\
\hline & Justificaciones & $0.44(0)$ & $1.11(1)$ & -2.230 & 0.026 & $1.44(1)$ & $2.00(2)$ & -1.633 & NS \\
\hline \multirow[t]{5}{*}{ Pollos } & Preguntas & 1.35 (1) & $1.10(1)$ & -1.890 & NS & $3.03(3)$ & $3.10(4)$ & -0.463 & NS \\
\hline & Pruebas & $1.29(1)$ & $1.35(1)$ & -0.263 & NS & $3.06(3)$ & 3.19 (3) & -0.623 & NS \\
\hline & Hipótesis & $0.61(0)$ & $0.45(0)$ & -1.000 & NS & $1.68(1)$ & $1.61(1)$ & -0.321 & NS \\
\hline & Conclusiones & 0.90 (1) & 0.94 (1) & -0.184 & NS & $2.26(2)$ & $2.84(3)$ & -2.190 & 0.029 \\
\hline & Justificaciones & $0.35(0)$ & $0.32(0)$ & -0.367 & NS & $1.42(1)$ & $1.32(1)$ & -0.732 & NS \\
\hline
\end{tabular}

Tabla 2. Respuestas adecuadas según los autores a las preguntas de la actividad. EP: experimento con pollos; EG: experimento con gatos; EE: experimento con el pez espinoso.

Fuente: Elaboración propia.

En la anterior tabla 2, se calculó la media (x), la mediana (Mdn), el valor de la prueba de Wilcoxon (Z) y la significación $(p)$, con la denominación (NS) cuando no se encuentran diferencias significativas y en negrita cuando presentan diferencias significativas.

De los resultados cabe destacar que no hubo diferencias significativas en cuanto a la cantidad de elementos identificados en el vídeo al responder en formato papel como en el propio vídeo con una anotación, a excepción del experimento del pez espinoso que presentó más cantidad de justificaciones cuando se realizó en el vídeo con Coannotation. Sin embargo, la calidad presentada en la explicación que realizaron los estudiantes sobre los elementos identificados fue mayor en las experiencias de los pollos y los gatos en las preguntas, pruebas y conclusiones, a excepción del experimento del pez espinoso que no produjo diferencias significativas en la calidad de las explicaciones. Uno de los elementos más difíciles de identificar fue la justificación y las conclusiones científicas, siendo estas partes de la argumentación científica de los investigadores del vídeo.

A continuación, se muestran ejemplos de estudiantes que mejoran cuando presentan una explicación en el vídeo que cuando lo hacen en papel para los elementos en los que hubo diferencias significativas con mejor evaluación (preguntas, pruebas y conclusiones).

Respuesta del estudiante 086V para la identificación de preguntas de investigación sobre la experiencia de los gatos (Figura 3 ). 


\section{Enumera todas las preguntas de investigación que se plantean en el vídeo}

a) - ¿cuál en la capacidad de menoria?

b)

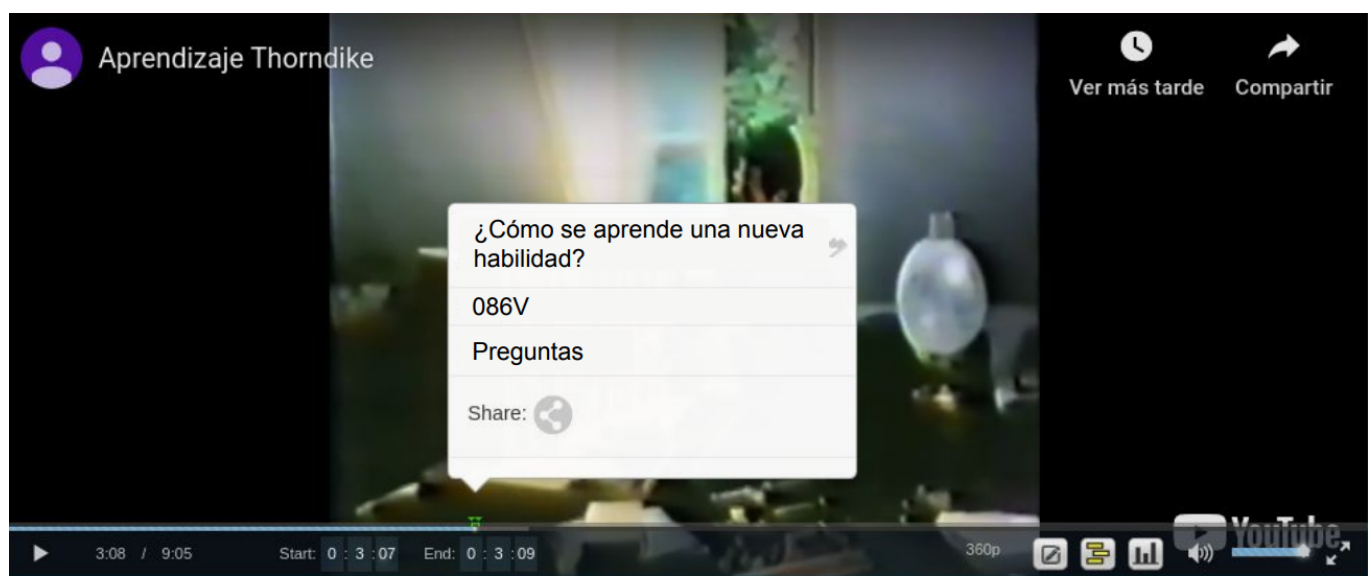

Figura 3. Identificación de una pregunta de investigación por el estudiante 086V: a) En coannotation.com y b) En papel

Fuente: Elaboración propia

En esta figura 3, el estudiante 086V explica cuál puede ser la pregunta de investigación que encuentra en la experiencia de los gatos de dos formas diferentes: en el caso de la respuesta en papel indica "¿Cuál es capacidad de memoria?" (figura 3a), que según la rúbrica de la figura 2 es considerado como nivel 2, porque aunque identifica una pregunta de investigación, esta es inadecuada; mientras que en el caso de la respuesta en forma de anotación sobre el vídeo (figura $3 b)$, indica "¿Cómo se aprende una nueva habilidad?", lo que se corresponde con el nivel 4 al ser adecuada.

Respuesta del estudiante 487X para la identificación de preguntas de investigación la experiencia de los gatos (Figura 4).

a)

\section{Enumera todas las pruebas de investigación que aparecen en el vídeo}
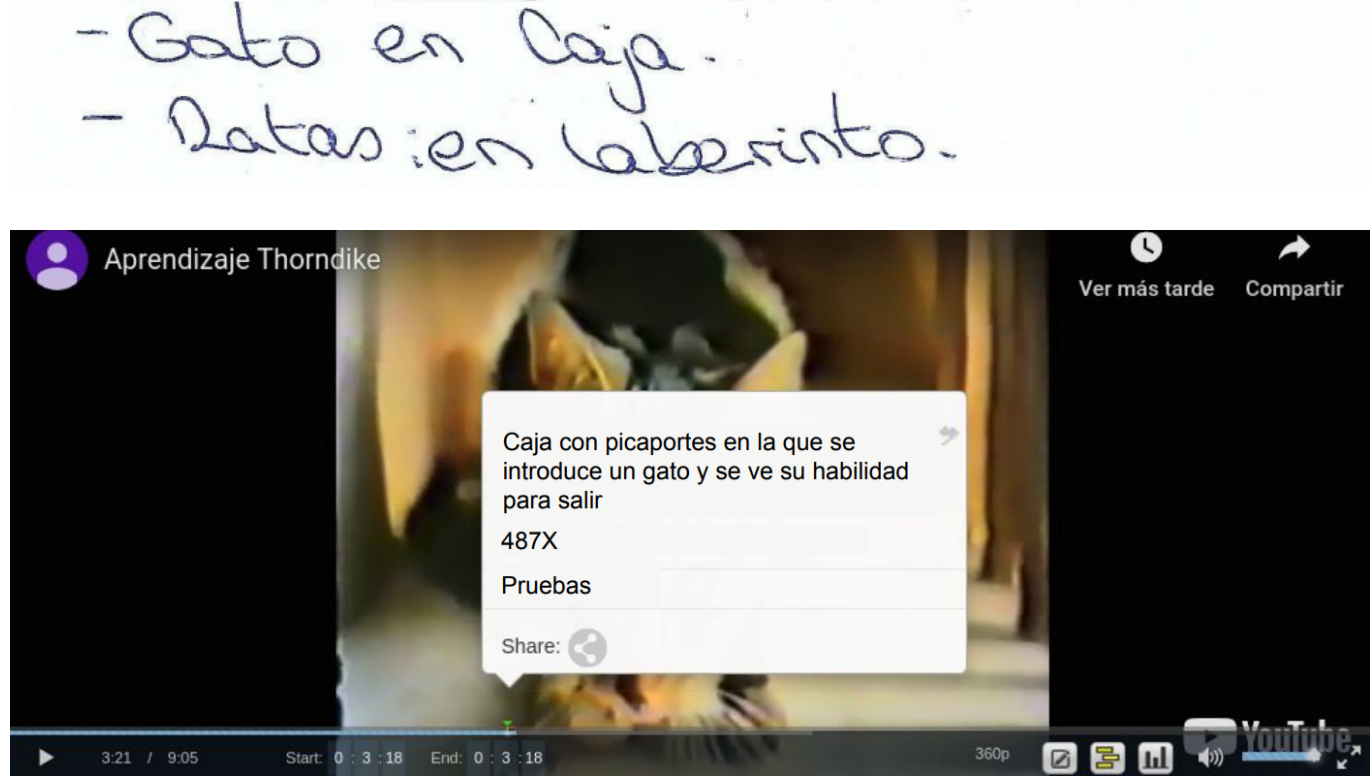
Figura 4. Identificación de una pregunta de investigación por el estudiante 599N: a) En coannotation.com y b) En papel

Fuente: Elaboración propia

En esta figura 4, el estudiante 487X aporta pruebas usadas en la investigación de los gatos de dos formas diferentes: en el caso de la respuesta en papel indica dos pruebas, el "Gato en caja" y las "Ratas en el laberinto" (figura 4a), que según la rúbrica de la figura 2 es considerado como nivel 2, porque identifica pruebas de investigación erróneas; mientras que en el caso de la respuesta en forma de anotación sobre el vídeo (figura 4b), indica una sóla prueba, pero es correcta, "Caja con picaportes en la que se introduce un gato y se ve su habilidad para salir", lo que se corresponde con el nivel 4 al ser adecuada.

Respuesta del estudiante 553E para la identificación de preguntas de investigación sobre la experiencia de los gatos (figura 5).

\section{Enumera todas las cónclusiones a las que llega el narrador del vídeo}

a)

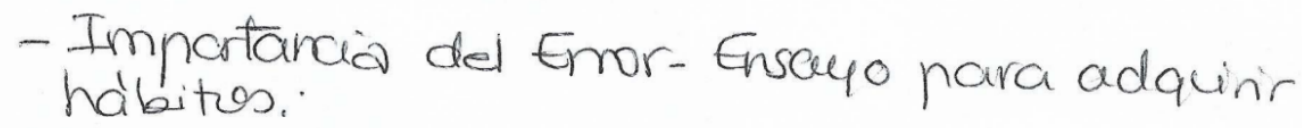

b)

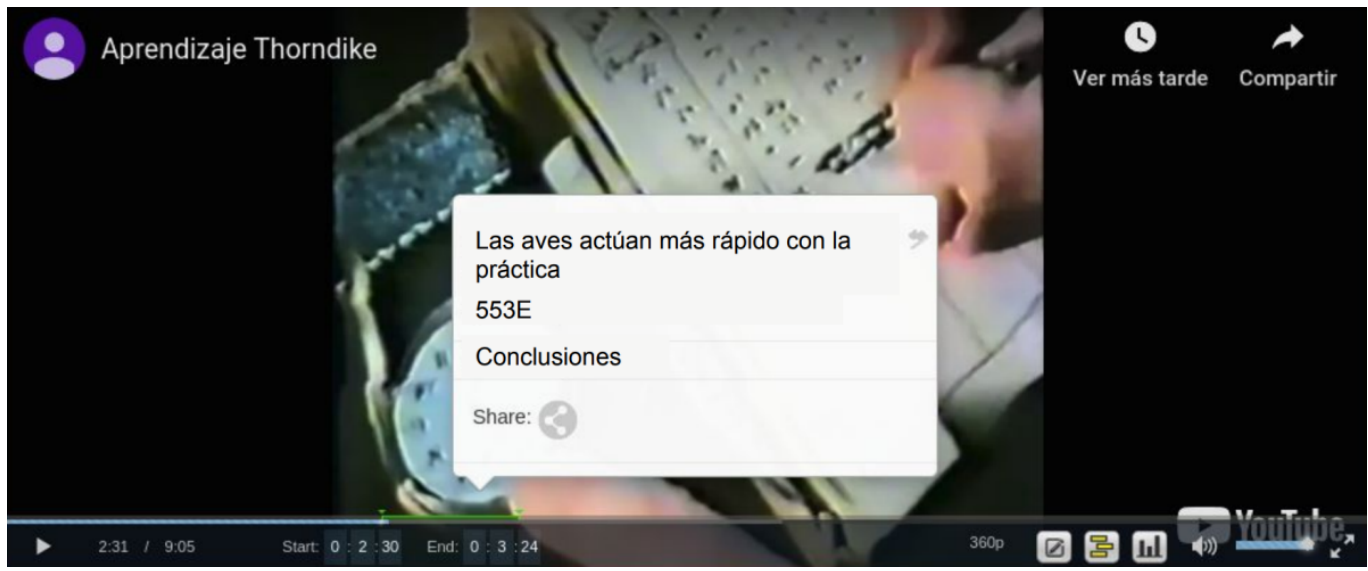

Figura 5. Identificación de una pregunta de investigación por el estudiante 553E: a) En coannotation.com y b) En papel

Fuente: Elaboración propia

En esta figura 5, el estudiante 553E aporta conclusiones de la investigación de los gatos de dos formas diferentes: en el caso de la respuesta en papel indica la "Importancia del error-ensayo para adquirir hábitos" (figura 5a), que según la rúbrica de la figura 2 es considerado como nivel 2, porque la conclusión de investigación es errónea; mientras que en el caso de la respuesta en forma de anotación sobre el vídeo (figura 5b), indica una sóla conclusión, pero es adecuada: "Las aves actúan más rápido con la práctica", lo que se corresponde con el nivel 4.

\section{Discusión}

Este trabajo muestra una investigación sobre una innovación en la enseñanza de las ciencias a través el vídeo, ya que representa un poderoso recurso educativo (Gaudin \& Chaliès, 2015), al mostrar experiencias narradas por investigadores reconocidos por la comunidad científica y que captan la atención del alumnado. A su vez en este trabajo, las anotaciones de vídeo desplaza los modelos en el aula, desde el énfasis en la transmisión del conocimiento del docente al alumno, 
hacia modelos que acentúan la construcción del conocimiento y su validación por medio de, entre otras, la identificación de los elementos de una investigación científica como paso previo a la investigación o indagación escolar, que es reconocida como una de las prácticas científicas más importantes (Driver, Newton, \& Osborne, 2000; Duschl \& Osborne, 2002). Estas nuevas orientaciones en el ámbito de la enseñanza de las ciencias (Keith Sawyer, 2006; Pellegrino, Chudowsky, \& Glaser, 2001) destacan porque los estudiantes son capaces de plantear formas complejas de razonamiento similares a las propias de la actividad científica. Por eso, este enfoque de identificación mediante anotaciones de vídeo abre nuevos espacios de conocimientos sobre el análisis de investigaciones científicas, donde se puedan justificar los aprendizajes desde una perspectiva dialogada, pensada y crítica.

En los resultados de la identificación de las justificaciones y conclusiones científicas se observa el bajo acierto, (en el primer nivel de logro de la rúbrica), que el alumnado tiene al identificar los elementos del argumento de los científicos implicados en el vídeo, fruto de la dificultad que encuentran los estudiantes para involucrarse en actividades de argumentación científica (Sampson \& Clark, 2008). El proceso de identificación, según el modelo de Toulmin (1958), de elementos de argumentos fomenta el pensamiento crítico argumentativo que, según Osborne et al (2016), es parte del proceso de argumentación y se considera como una capacidad distinta a la producción y construcción de un argumento.

\section{Conclusiones}

En este trabajo se ha partido de la idea de que antes de formar en la competencia de indagación y experimentación para mejorar las prácticas docentes de los estudiantes de educación, es necesario capacitarlos conceptualmente sobre cómo realizar una indagación desde procedimientos científicos (van Uum et al., 2016). De esta manera, se pueden mitigar las dificultades que suelen presentar los estudiantes de educación en el proceso de diseño de actividades de indagación y experimentación para mejorar sus prácticas (Windschitl, 2003). Por tanto, partiendo de la premisa de que las anotaciones sobre los vídeos suponen un impacto positivo en el aprendizaje de la indagación y experimentación científica, se ha comprobado que permiten que el alumnado se ejercite en la identificación de elementos que componen una investigación científica, que tanto trabajo supone (Vílchez-González \& Bravo-Torija, 2015), como paso previo para realizar una indagación escolar.

Los resultados muestran que no hay diferencias significativas en cuanto a la misma cantidad de respuestas según los dos formatos. No obstante, sí hubo diferencias significativas en cuanto a la calidad o aciertos y adecuación del tipo de respuestas cuando se realizaron con anotaciones sobre el video. Siendo significativas en tres de los cinco elementos que se consideraron, como fueron: las preguntas, pruebas y conclusiones de las investigaciones. Encontrando dos posibles motivos de estas diferencias que pueden deberse a: la repetida visualización para seleccionar un fragmento del vídeo cuando se utilizan las anotaciones de vídeo, mientras que para el formato de papel pudiera parecer que no es tan necesario visualizar una y otra vez el vídeo; así como, la obligatoriedad de tomar notas en papel mientras se proyecta el vídeo en la clase, lo que puede provocar que se pierdan partes importantes de información mientras se escribe. Con las anotaciones de vídeo, no es necesario que exista esta toma de notas mediante la proyección, ya que se puede visualizar el vídeo a la vez que se va subrayando.

La identificación de pregunta pueden ser complicada ya que son parafraseadas en el vídeo, y hay que prestar especial atención para identificarlas. Por ello, el hacerlo en formato de anotación de vídeo puede favorecer la comprensión de esta. En las experiencias científicas se presentan múltiples pruebas que apoyan a la pregunta de investigación, pero si ya existe una dificultad para 
identificar la pregunta, las pruebas también presentarán una dificultad añadida. Por último, la identificación de las justificaciones de una investigación científica necesita de la pregunta y las pruebas para hacer de nexo de unión y, por ello, ocurre lo mismo que con las pruebas. No obstante, son más fácil de comprenderlas cuando se hace una evaluación mediante anotaciones de vídeo. Del resto de elementos (las hipótesis y las justificaciones), no tenemos pruebas suficientes para afirmar que haya diferencia entre el formato de evaluación mediante texto o anotación de vídeo y, por tanto, que se mejore la identificación y el aprendizaje de estos a través de las anotaciones de vídeo.

Se hace necesaria una comparación con otros formatos de evaluación de los elementos de investigaciones como pudieran ser evaluaciones en formato oral, y otras investigaciones que ratifiquen estos resultados para otros contextos diferentes, como pudieran ser, la identificación de otros contenidos didácticos.

\section{Agradecimientos}

Este trabajo forma parte del proyecto de I+D de Excelencia "Desarrollo de competencias en problemas de la vida diaria mediante prácticas científicas de argumentación, indagación y modelización en enseñanza secundaria y universitaria" (EDU2017-82197-P), financiado por el Ministerio de Economía, Industria y Competitividad en 2017"

\section{Referencias}

Alonso-Ferreiro, A. (2018). Aprendizaje basado en proyectos para el desarrollo de la competencia digital docente en la formación inicial del profesorado. RELATEC: Revista Latinoamericana de Tecnología Educativa, 17(1), 9-24.

Barth-Cohen, L. A., Little, A. J., \& Abrahamson, D. (2018). Building reflective practices in a pre-service math and science teacher education course that focuses on qualitative video analysis. Journal of Science Teacher Education, 29(2), 83-101.

Bozdag, A. D. (2008). A new technique for presentation of scientific works: video in poster. World Journal of Surgery, 32(7), 1559-1561.

Bryan, L. A., \& Recesso, A. (2006). Promoting reflection among science student teachers using a web-based video analysis tool. Journal of Computing in Teacher Education, 23(1), 31-39.

Cabero-Almenara, J., Roig-Vila, R., \& Mengual-Andrés, S. (2018). Conocimientos tecnológicos, pedagógicos y disciplinares de los futuros docentes según el modelo TPACK. Digital Education Review, (32), 73-84.

Castaño-Garrido, C., Garay-Ruiz, U., \& Themistokleous, S. (2018). De la revolución del software a la del hardware en educación superior. RIED. Revista Iberoamericana de Educación a Distancia, 21(1), 135153.

Cebrián-de-la-Serna, M., Bartolomé-Pina, A., Cebrián-Robles, D., \& Ruiz-Torres, M. (2015). Estudio de los Portafolios en el Practicum: Análisis de un PLE-Portafolios. Revista Electrónica de Investigación y Evaluación Educativa, 21(2), 1-18.

Cebrián-Robles, D., Blanco-López, Á., \& Noguera-Valdemar, J. (2016). El uso de anotaciones sobre vídeos en abierto como herramienta para analizar las concepciones de los estudiantes de pedagogía sobre un problema ambiental. CIDTFF - Indagatio Didactica, 8(1), 158-174.

Cebrián-Robles, D., Cebrián-de-la-Serna, M., \& Monedero-Moya, J.-J. (2015). Study of video annotations in external practices of university learning. conference: ECER 2015. Budapest: ECER. Retrieved from http://hdl.handle.net/10630/10240

Cebrián-Robles, D., Franco-Mariscal, A.-J., \& Blanco-López, Á. (2018). Preservice Elementary Science Teachers' Argumentation Competence: Impact of a Training Programme. Instructional Science, 46(5), 789-817. 
Cela-Ranilla, J. M., Esteve González, V., Esteve Mon, F., González Martínez, J., \& Gisbert-Cervera, M. (2017). El docente en la sociedad digital: una propuesta basada en la pedagogía transformativa y en la tecnología avanzada. Profesorado. Revista de Currículum Y Formación de Profesorado, 21(1), 403-422.

Chiu, P.-S., Chen, H.-C., Huang, Y.-M., Liu, C.-J., Liu, M.-C., \& Shen, M.-H. (2016). A video annotation learning approach to improve the effects of video learning. Innovations in Education and Teaching International, 55(4) 459-469.

Dovigo, F. (2016). Argumentation in preschool: a common ground for collaborative learning in early childhood. European Early Childhood Education Research Journal, 24(6), 818-840.

Driver, R., Newton, P., \& Osborne, J. (2000). Establishing the norms of scientific argumentation in classrooms. Science Education, 84(3), 287-312.

Duschl, R. A., \& Osborne, J. (2002). Supporting and promoting argumentation discourse in science education. Studies in Science Education, 38(1), 39-72.

Forbes, C. T., \& Davis, E. A. (2010). Curriculum design for inquiry: Preservice elementary teachers' mobilization and adaptation of science curriculum materials. Journal of Research in Science Teaching, 47(7), 820839.

García-Ruiz, R., Aguaded, I., \& Bartolomé-Pina, A. (2018). La revolución del blended learning en la educación a distancia. RIED. Revista Iberoamericana de Educación a Distancia, 21(1), 25-32.

García-Valcárcel, A., \& Martín-del-Pozo, M. (2016). Análisis de las competencias digitales de los graduados en titulaciones de maestro. RELATEC, 15(2), 155-168.

Gaudin, C., \& Chaliès, S. (2015). Video viewing in teacher education and professional development: A literature review. Educational Research Review, 16, 41-67.

Hernandez-Ramos, P. (2007). Aim, shoot, ready! Future teachers learn to do video. British Journal of Educational Technology: Journal of the Council for Educational Technology, 38(1), 33-41.

Jiménez-Aleixandre, M. P. (2010). 10 Ideas Clave. Competencias en argumentación y uso de pruebas (Vol. 12). Barcelona: Graó.

Jiménez-Aleixandre, M. P., Rodriguez, A. B., \& Duschl, R. A. (2000). "Doing the lesson" or" doing science": Argument in high school genetics. Science Education, 84(6), 757-792.

Keith Sawyer, R. (2006). The Cambridge Handbook of the Learning Sciences. Cambridge University Press.

Marqués, A. R., \& Reis, P. (2017). Producción y difusión de vídeos digitales sobre contaminación ambiental. Estudio de caso: Activismo colectivo basado en la investigación. Revista Eureka sobre enseñanza y divulgación de las ciencias, 14(1), 215-226.

Minner, D. D., Levy, A. J., \& Century, J. (2010). Inquiry-based science instruction-what is it and does it matter? Results from a research synthesis years 1984 to 2002. Journal of Research in Science Teaching, 47(4), 474-496.

Mirriahi, N., Jovanovic, J., Dawson, S., Gašević, D., \& Pardo, A. (2018). Identifying engagement patterns with video annotation activities: A case study in professional development. Australasian Journal of Educational Technology, 34(1), 57-72.

Montero, L., \& Gewerc, A. (2018). La profesión docente en la sociedad del conocimiento. Una mirada a través de la revisión de investigaciones de los últimos 10 años. Revista de Educación a Distancia, (56), 2-22.

Osborne, J., Erduran, S., \& Simon, S. (2004). Ideas, evidence \& argument in science. Londres: King's College.

Osborne, J., Henderson, J. B., MacPherson, A., Szu, E., Wild, A., \& Yao, S. (2016). The development and validation of a learning progression for argumentation in science. Journal of Research in Science Teaching, 53(6), 821-846.

Pellegrino, J. W., Chudowsky, N., \& Glaser, R. (2001). Knowing What Students Know (Vol. 19). Washington, DC: National Academy Press. 
Sadler, T. D. (2011). Situating Socio-scientific issues in classrooms as a means of achieving goals of science education. In T. D. Sadler (Ed.), Socio-scientific Issues in the Classroom: Teaching, Learning and Research (pp. 1-9). New York: Springer.

Sampson, V., \& Clark, D. B. (2008). Assessment of the ways students generate arguments in science education: Current perspectives and recommendations for future directions. Science Education, 92(3), 447-472.

Santagata, R., \& Guarino, J. (2011). Using video to teach future teachers to learn from teaching. ZDM: The International Journal on Mathematics Education, 43(1), 133-145.

Thorndike, E. L. (1898). Animal intelligence: An experimental study of the associative processes in animals. The Psychological Review: Monograph Supplements, 2(4). Retrieved from https://bit.ly/2UajiXP

Tinbergen, N. (1952). The Curious Behavior of the Stickleback.Scientific American, 187(6), 22-27.

Toma, R. B., Greca, I. M., \& Meneses-Villagrá, J. Á. (2017). Dificultades de maestros en formación inicial para diseñar unidades didácticas usando la metodología de indagación. Revista Eureka sobre enseñanza y divulgación de las ciencias, 14(2), 442-457.

Toulmin, S. E. (1958). The uses of argument (2003rd ed.). Cambridge: Cambridge University Press.

van Uum, M. S. J., Verhoeff, R. P., \& Peeters, M. (2016). Inquiry-based science education: towards a pedagogical framework for primary school teachers.International Journal of Science Education, 38(3), 450-469.

Vega, S. (2011). Ciencia 3-6: Laboratorios de ciencias en la escuela infantil. Barcelona: Grao.

Vílchez-González, J.-M., \& Bravo-Torija, B. (2015). Percepción del profesorado de ciencias de educación primaria en formación acerca de las etapas y acciones necesarias para realizar una indagación escolar. Enseñanza de las ciencias, 33(1), 0185-0202.

Windschitl, M. (2003). Inquiry projects in science teacher education: What can investigative experiences reveal about teacher thinking and eventual classroom practice? Science Education, 87(1), 112-143. 